\title{
World Oral Health Day 2021
}

\author{
Francisco Rodriguez Lozano
}

World Oral Health day, on 20 March, is a day to promote the important contribution of oral health to overall health. We should always keep in mind the association of oral pathology and diseases in different parts of the body. As president of the ENSP, I would like to take the opportunity on this special day to highlight the close relationship between tobacco use and oral health ${ }^{1,2}$.

Tobacco in all forms and the associated nicotine are harmful to the body, causing damage to our health from the oral cavity to the lungs, bladder, and the reproductive system, to mention only a few of the most common pathologies associated with the use of both e-cigarettes and cigarettes. The oral cavity is the route through which nicotine and tobacco usually enter our body; it is precisely here where significant damage begins.

The deleterious effects of tobacco use on the oral cavity vary greatly. They can range from staining of teeth, which can be reversed, to oral cancer which in Europe has a $50 \%$ mortality rate mainly due to delayed diagnosis. Other consequences are halitosis or bad breath, loss of taste or smell, and more importantly a delay in wound-healing with all the ensuing complications for dental interventions ${ }^{1,2}$.

The most frequent condition caused by tobacco is periodontal disease. The different types worsen the prognosis after surgery and lead to poorer results, increasing the number of implant placement failures. Refractory periodontitis is another type of periodontitis that causes continued attachment loss in spite of adequate treatment and proper oral hygiene. Some other pathologies frequently linked to tobacco use are oral candidiasis, palatal nicotinic stomatitis and the so-called 'smoker's melanosis'. Compared to smokers of conventional cigarettes, smokers of e-cigarettes present a higher rate of nicotine stomatitis, hyperplastic candidiasis and 'black hairy' tongue ${ }^{3-8}$. The precancerous lesions found in smokers should also be mentioned. These are lesions that can develop into cancerous ones. Hence, the importance of prevention, early detection and treatment cannot be emphasized enough.

All the above facts have made most dental professionals highly aware of their importance in giving advice to patients on smoking cessation, helping them to quit and preventing patients from starting tobacco or nicotine use ${ }^{1,9}$.

Dental professionals are becoming more involved in the daily struggle against the tobacco industry in order to improve the health of their patients and that of the general population. In this way they are aligned with the rest of the health professionals, non-governmental organizations and Health Departments in the fight against an agent that in Europe alone annually kills 0.7 million people with a concomitant daily healthcare burden of $€ 65$ million, not to mention the pain and long suffering experienced by smokers and their families.
AFFILIATION

1 European Network for Smoking and Tobacco Prevention, Brussels, Belgium

CORRESPONDENCE TO Francisco Rodriguez Lozano. European Network for Smoking and Tobacco Prevention, Chaussée d'Ixelles 144, 1050 Brussels, Belgium. E-mail: frguez@infomed.es

\section{KEYWORDS}

smoking, tobacco, oral health, vaping, e-cigarettes

Received: 12 March 2021 Accepted: 16 March 2021 


\section{REFERENCES}

1. ENSP initiative on Oral Health and Tobacco. European Network for Smoking and Tobacco Prevention webinar. March 19, 2021. Accessed March 19, 2021. http://ensp. network/19-march-webinar-ensp-initiative-on-oralhealth-and-tobacco/

2. Zhang Y, He J, He B, Huang R, Li M. Effect of tobacco on periodontal disease and oral cancer. Tob Induc Dis. 2019;17(May):1-15. doi:10.18332/tid/106187

3. Asthana S, Vohra P, Labani S. Association of smokeless tobacco with oral cancer: A review of systematic reviews. Tob Prev Cessat. 2019;5(October):1-12. doi:10.18332/tpc/112596

4. Ebersole J, Samburova V, Son Y, Cappelli D, Demopoulos C, Capurro A, et al. Harmful chemicals emitted from electronic cigarettes and potential deleterious effects in the oral cavity. Tob Induc Dis. 2020;18(May):1-16. doi:10.18332/tid/116988

5. Irusa KF, Vence B, Donovan T. Potential oral health effects of e-cigarettes and vaping: A review and case reports. J Esthet Restor Dent. 2020;32(3):260-264. doi:10.1111/jerd.12583

6. Akinkugbe AA. Cigarettes, E-cigarettes, and Adolescents' Oral Health: Findings from the Population Assessment of Tobacco and Health (PATH) Study. JDR Clin Trans Res. 2019;4(3):276-283. doi:10.1177/2380084418806870

7. Rouabhia M. Impact of Electronic Cigarettes on Oral Health: a Review. J Can Dent Assoc. 2020;86:k7. Accessed March 12, 2021. https://jcda.ca/k7

8. Yang I, Sandeep S, Rodriguez J. The oral health impact of electronic cigarette use: a systematic review. Crit Rev Toxicol. 2020;50(2):97-127. doi:10.1080/10408444.2020.1713726

9. Holliday R, Hong B, McColl E, Livingstone-Banks J, Preshaw PM. Interventions for tobacco cessation delivered by dental professionals. Cochrane Database Syst Rev. 2021;2:CD005084. doi:10.1002/14651858.CD005084.pub4
CONFLICTS OF INTEREST

The author has completed and submitted the ICMJE Form for Disclosure of Potential Conflicts of Interest and none was reported.

FUNDING

There was no source of funding for this research.

PROVENANCE AND PEER REVIEW

Commissioned; internally peer reviewed. 